$$
\begin{gathered}
\text { 주의력결핍 과잉행동장애 환아 평가시 지능검사의 } \\
\text { 유용성에 관한 예비연구 }
\end{gathered}
$$

민정원 · 이원혜 · 홍민하 · 반건호

경희대학교 의학전문대학원 정신건강의학교실

\title{
A Pilot Study of the Usefulness of Intelligence Test in Assessment of Attention-Deficit Hyperactivity Disorder
}

\author{
Jung-Won Min, M.D., Won-Hye Lee, Ph.D., Min-Ha Hong, M.D. and Geon Ho Bahn, M.D., Ph.D. \\ Department of Psychiatry, Kyung Hee University School of Medicine, Seoul, Korea
}

Objectives : We analyzed the usefulness of intelligence test in assessing attention-deficit hyperactivity disorder (ADHD).

Methods : The medical records of 312 patients with ADHD who visited the child psychiatry department in one university hospital in Seoul, Korea, were reviewed. Demographic data and scores on the Korean Wechsler Intelligence Scale for Children-III (KWISC-III) and Korean-Conners' Parent Rating Scale (K-CPRS) were analyzed. To assess the relationship with comorbidities, ADHD subjects were classified as; externalizing disorders, internalizing disorders, tic disorders, and no comorbidities.

Results : Verbal intelligence quotient (VIQ) was significantly higher than performance IQ (PIQ)(102.6 $\pm 14.44,99.2 \pm 14.48$, $\mathrm{p}<.001$ ). Using mean scatter method, subtest scores such as 'information, similarities, digit span and coding' were significantly lower than mean scores of sum of subtests of VIQ or PIQ $(\mathrm{p}<.05)$. Regarding comorbidities, children with externalizing disorders exhibited. Lower scores on 'information and vocabulary' than other groups ( $p=.008)$. Children with no comorbidities exhibited higher scores on 'similarities and object assembly' than children with externalizing/internalizing disorders $(p=.001)$ and also on 'comprehension' than children with externalizing disorders ( $\mathrm{p}=.006)$. For subtypes of ADHD, children with hyperactive-impulsive type had higher scores on 'comprehension' than children with inattentive and combined type ( $\mathrm{p}=.004)$.

Conclusion: These results suggested that intelligence test might provide useful information for assessing ADHD.

KEY WORDS : ADHD $\cdot$ Intelligence Test $\cdot$ Comorbidity $\cdot$ Subtype.

\section{서 론}

주의력결핍 과잉행동장애(attention-deficit hyperactivity disorder, ADHD)는 임상 면담과 관찰, 부모와 교사의 보고, 임상 평가 도구 등으로 진단한다. ${ }^{1,2)} \mathrm{ADHD}$ 환자의 심리검사 는 표준화된 지능검사와 학습능력검사로 구성되며, 그 외에도 신경심리검사, 언어평가, 주의력 및 억제조절을 보는 전산화 검

접수완료:2012년 11월 23일 / 수정완료:2012년 12월 10일 심사완료:2012년 12월 14일

Address for correspondence:Geon Ho Bahn, M.D., Ph.D., Department of Psychiatry, Kyung Hee University School of Medicine, 23 Kyungheedae-ro, Dongdaemungu, Seoul 130-872, Korea

Tel : +82.2-2-958-8556, Fax : +82.2-957-1997

E-mail : mompeian@khu.ac.kr

이 논문의 주제는 2012년 7월 25일 제20차 IACAPAP, Paris에서 포스터 발표하였음.
사 등이 포함된다. ${ }^{3)}$

지능검사를 통해 $\mathrm{ADHD}$ 와 지적장애를 변별할 수 있고 학 습장애를 감별할 수 있을 뿐만 아니라, 검사를 하는 동안 환아 의 행동을 관찰함으로써 검사 결과의 유효성을 판정하고 증상 을 감지해낼 수가 있다는 것이 그 이유이다.") Bowers 등5)은 Wechsler's Deterioration Index(WDI)를 통해 ADHD 아동과 $\mathrm{ADHD}$ 가 아닌 아동을 변별할 수 있으며, WDI가 의미 있게 증 가하였을 때 $\mathrm{ADHD}$ 진단 가능성을 고려하여야 한다고 주장 하였으며, 대표적 지능검사인 Wechsler Intelligence Scale for Children-III(WISC-III)의 네 가지 소검사, 즉 숫자, 상식, 어 휘, 차례맞추기를 $\mathrm{ADHD}$ 진단시 예측인자로 사용할 수 있음 을 제안하는 연구도 있었다. ${ }^{6}$ Prifitera와 Saklofske ${ }^{7)}$ 는 이전 연구들을 요약하면서 $\mathrm{ADHD}$ 환아에서 주의집중과 처리속도 등 두 요인지표의 점수가 낮으며, 소검사와 관련하여 이 요인지 
표에 해당하는 기호쓰기, 산수, 동형찾기, 숫자 등의 점수가 낮 음을 보고하였다.

그럼에도 불구하고 $\mathrm{ADHD}$ 진단 보조 도구로서 지능검사의 유용성에 대한 논란은 계속되고 있다. ${ }^{7)}$ Prifitera와 Dersh ${ }^{8}$ 는 $\mathrm{ADHD}$ 환아의 지능검사 결과를 개개인 수준에서 분석하였을 때 주의집중과 처리속도 요인지표에 해당하는 소검사의 점수 에서 유의한 차이가 없다고 보고하였다. Kaufman과 Lichtenberger ${ }^{9}$ 도 ADHD 환아들에게 있어 WISC-III 검사가 아이들 의 지적 능력과 인지적인 강점/약점에 대한 유용한 정보를 제 공할 수는 있지만, 소검사 수행과 관련된 전반적인 패턴이 각 각의 환아들에게 반드시 나타나리라는 법은 없으며, WISC$\mathrm{III}^{10)}$ 는 $\mathrm{ADHD}$ 에 대한 다양한 평가 도구 중 일부로만 사용되 어야 한다고 주장하였다. Cohen 등1ㅡㄴ 즌 지능검사의 주의집중 요인이 부모나 선생님의 보고와 일치하지 않는다고 보고하였 으며, 주의집중 요인으로 $\mathrm{ADHD}$ 와 다른 질환들을 감별할 수 없다는 연구도 있었다.

지능검사에 소요되는 비용과 검사 시간을 감안할 때, $\mathrm{ADHD}$ 평가시 지능검사를 포함시켜야 하는가에 대한 심도 있는 논의 가 필요하다. 이에 본 저자들은 국내 일 대학병원에서의 $\mathrm{ADHD}$ 환아들의 지능검사 결과를 분석하여 $\mathrm{ADHD}$ 평가시 지능검사 의 유용성 여부에 대해 알아보았다.

\section{방 법}

\section{1. 대 상}

2005년 3월부터 2012년 2월까지 국내 일 대학병원의 소아 청소년 정신건강의학과에서 정신장애의 진단 및 통계 편람 제 4판(Diagnostic and Statistical Manual of Mental Disorders $4^{\text {th }}$ edition Text Revision version, DSM-IV-TR)에 의거하여 $\mathrm{ADHD}$ 로 진단받은 만 6세에서 만 16 세까지의 환아 312 명의 진료기록과 심리평가 보고서를 후향적으로 조사하였다. 사회 인구학적 변인에는 성별과 연령이 포함되었다.

지능검사 특성을 보기 위하여 한국판 웩슬러 아동용 지능 검사-3판의 전체지능, 언어성 지능, 동작성 지능 및 각 소검사 를 분석하였다. $\mathrm{ADHD}$ 와 관련된 행동 증상을 평가하기 위해 단축형 코너스 부모용 평정척도를 분석하였다.

공존질환이 $\mathrm{ADHD}$ 와 지능검사에 미치는 영향을 알아보기 위하여 공존질환 없는 군, 내재화 장애(internalizing disorder) 집단, 외현화 장애(externalizing disorder) 집단, 틱장애 집단 등 대상군을 네 군으로 분류하였다. Takeda 등 ${ }^{12)}$ 의 $\mathrm{ADHD}$ 공 존질환 유무에 따른 비교연구에서는 공존질환을 내재화/외 현화 장애로 분류하였으나, 본 연구에서는 틱장애가 인지기 능에 영향을 주지 않는다는 Roessner 등 ${ }^{13)}$ 의 연구 결과를 확
인해보고자 틱장애 집단을 별도로 구분하였다.

외현화 장애 집단에는 품행장애 및 적대적 반항장애가 포 함되었고, 불안장애, 우울장애, 적응장애는 내재화 장애로 분 류하였다. 공존질환의 진단은 DSM-IV-TR의 기준에 따랐다. 공존질환 중 지능검사에 미치는 영향을 고려하여 발달장애, 조현병, 간질, 지적장애가 공존하는 경우는 연구대상에서 배제 하였다. 항정신병 약물은 인지기능에 영향을 미칠 수 있으므 로 $^{14}$ 틱장애 집단 중 항정신병 약물을 사용하고 있는 환아들 도 연구대상에서 제외하였다. 그 밖에 유뇨증(6명), 양극성 장 애(4명), 학습장애(3명), 의사소통장애(3명), 식이장애(3명), 충 동조절장애(2명), 반응성 애착장애(1명), 유분증(1명)의 공존질 환이 있었으나 통계적 의의를 고려하여 공존질환에 따른 지능 지수와의 관련 비교에서는 제외하였다.

$\mathrm{ADHD}$ 아형은 DSM-IV-TR에 의거하여 부주의형, 과잉행 동-충동성형, 복합형으로 분류하였다.

\section{2. 척 도}

\section{1) 한국판 웩슬러 아동용 지능검사-3판(Korean Wechsler Intelligence Scale for Children-III, K-WISC-III)}

$\mathrm{K}-\mathrm{WISC}-\mathrm{III}^{15)}$ 는 웩슬러 아동용 지능검사-3판 ${ }^{16)}$ 을 한국어 로 번안해 표준화한 개인용 지능검사로, 만 6세에서부터 만 17 세 미만의 아동 및 청소년의 지능을 임상적으로 평가할 수 있다. K-WSIC-III는 상식, 산수, 공통성, 어휘, 이해 및 숫자 (선택검사)의 여섯 가지 언어성 소검사와 빠진 곳 찾기, 차례 맞추기, 토막짜기, 모양 맞추기, 기호쓰기, 동형 찾기(선택검사), 미로(선택검사)의 일곱 가지 동작성 소검사로 구성되어 있으며, 전체지능, 언어성 지능, 동작성 지능의 세 가지 지능지수 외에 언어적 이해력 지표, 주의집중 지표, 지각적 추론 지표, 처리속 도 지표 등 네 개의 지표점수가 산출된다. 본 연구에서는 부록 검사인 동형 찾기와 미로검사를 제외한 11 가지 소검사 결과 를 채택하였으며, 각 소검사의 연령별 환산점수와 세 가지 지 능지수를 사용하였다.

\section{2) 단축형 코너스 부모용 평정척도(Short Form Korean- Conners' Parent Rating Scale, K-CPRS)}

코너스 평정척도 ${ }^{17)}$ 는 $\mathrm{ADHD}$ 와 관련된 행동 증상을 객관적 으로 평가하기 위하여 개발된 척도로, 부모용과 교사용 각각 에 대해 10 문항 단축형에서부터 98 문항까지 다양한 척도가 개발되어 있다. 본 연구에서는 국내에서 널리 사용되는 10 문 항의 단축형 $\mathrm{K}-\mathrm{CPRS}^{18)}$ 를 실시하였다. 각 문항은 '전혀 아니 다(0점)'부터 '매우 많이 그렇다(3점)'의 4점 척도로 평정하도 록 되어 있으며 점수가 높을수록 증상이 심한 것을 의미한다. 
준거 점수(cut-off value)는 16점 이상이다.

\section{3. 통계분석}

모든 통계는 SPSS 12.0(SPSS Inc., Chicago, IL, USA) 프 로그램을 사용하여 분석하였다.

환아들의 지능 편차를 살펴보기 위해 소검사 평균 점수를 기 준으로 각 소검사의 이탈 정도를 살펴보는 평균치 분산(mean scatter) 방법을 사용하였다. ${ }^{19)}$ 언어성 지능과 동작성 지능 간 의 차이, 언어성 소검사의 연령 환산점수 평균과 각 언어성 소 검사 점수 간의 차이, 동작성 소검사의 연령 환산점수 평균과 각 동작성 소검사 점수 간의 차이에 대해 Paired samples ttest를 실시하였다.

코너스 부모용 평정척도와 지능검사 항목 간의 관련성을 살 펴보고자 pearson correlation을 실시하였다.

공존질환에 따른 인지적 특성을 살펴보기 위해, 공존질환 유형에 따라 분류한 네 집단의 세 가지 지능지수와 11 가지 소 검사 점수 및 Conners' Parent Rating Scale(CPRS)의 차이에 대해서는 네 집단 간 유의한 차이를 보인 연령을 공변량으로 하여 다변량공산분석(multivariate analysis of covariance, MANCOVA)을 실시하였으며, 사후검증으로는 최소유의차검 정(least significant differences)을 실시하였다.

$\mathrm{ADHD}$ 아형에 따른 지능검사 특성의 비교를 위해 세 가지 지능지수와 11 가지 소검사 점수 및 $\mathrm{CPRS}$ 의 차이에 대해서는 다변량분산분석(multivariate analysis of variance, MANO$\mathrm{VA}$ )을 실시하였으며, 사후검증으로는 최소유의차검정(least significant differences)을 실시하였다. 각 분석의 유의수준은 0.05 이하로 하였다.

모든 분석 과정에서 각 소검사 점수의 합산 점수가 적용되 는 전체지능, 언어성 지능, 동작성 지능에 대해서는 Bonferroni 보정(Bonferroni correction)을 적용하였다.

\section{결 과}

연구 대상은 총 312명으로, 남자 환아가 257명(82.4\%)이었 으며, 연령의 범주는 만 6 세에서 16 세까지였고, 검사를 시행한 평균연령은 만 9.7 \pm 2.5 세였다(Table 1).

평균 전체지능은 $101.3 \pm 14.0$, 언어성 지능은 $102.6 \pm 14.4$, 동 작성 지능은 $99.2 \pm 14.5$ 였다. K-CPRS는 $14.0 \pm 6.3$ 점이었다. 공 존질환이 없는 경우가 198명, 공존질환이 있는 경우는 114명이 었다. 공존질환이 있는 군은 내재화 장애가 40 명, 외현화 장애 가 17 명, 틱장애가 34 명이었고, 기타 질환은 23명이었다. 아형 분류가 가능한 경우는 225명이었으며, 복합형(combined type) 이 124명, 부주의형(predominantly inattentive type)이 68명,
Table 1. Demographic and clinical characteristics of subjects

\begin{tabular}{|c|c|}
\hline & $\mathrm{N}(\%)$ or mean $\pm \mathrm{SD}$ \\
\hline \multicolumn{2}{|l|}{ Demographic data $(\mathrm{N}=312)$} \\
\hline Gender (male) & $257(82.4)$ \\
\hline Age (years) & $9.7 \pm 2.5$ \\
\hline \multicolumn{2}{|l|}{ K-WISC-III } \\
\hline $\mathrm{FSIQ}$ & $101.3 \pm 14.0$ \\
\hline $\mathrm{VIQ}$ & $102.6 \pm 14.4$ \\
\hline$P I Q$ & $99.2 \pm 14.5$ \\
\hline K-CPRS & $14.0 \pm 6.3$ \\
\hline \multicolumn{2}{|l|}{ Comorbidity (N=312) } \\
\hline No comorbidity & $198(63.5)$ \\
\hline Internalizing disorder & $40(12.8)$ \\
\hline Depressive disorder & 21 \\
\hline Anxiety disorder & 6 \\
\hline Adjustment disorder & 13 \\
\hline Externalizing disorder & $17(5.4)$ \\
\hline Oppositional defiant disorder & 8 \\
\hline Conduct disorder & 9 \\
\hline Tic disorder & $34(10.9)$ \\
\hline Etc & $23(7.4)$ \\
\hline Enuresis & 6 \\
\hline Bipolar disorder & 4 \\
\hline Learning disorder & 3 \\
\hline Language disorder & 3 \\
\hline Eating disorder & 3 \\
\hline Impulse control disorder & 2 \\
\hline Reactive attachment disorder & 1 \\
\hline Encopresis & 1 \\
\hline \multicolumn{2}{|l|}{ ADHD subtype $(\mathrm{N}=225)$} \\
\hline Combined type & $124(55.1)$ \\
\hline Inattentive type & $68(30.2)$ \\
\hline Hyperactive-impulsive type & $33(14.7)$ \\
\hline
\end{tabular}

K-WISC-III : Korean Wechsler Intelligence Scale for Children-III, SD : standard deviation, FSIQ : Full Scale IQ, VIQ : verbal IQ, PIQ : performance IQ, K-CPRS : Korean-Conners' Parent Rating Scale, AD$H D$ : attention-deficit hyperactivity disorder, $I Q$ : intelligence quotient

과잉행동-충동성형(predominantly hyperactive-impulsive type)이 33명이었다.

언어성 지능은 동작성 지능보다 통계적으로 유의미하게 높 았다(t=4.226, $\mathrm{p}<.001$ )(Table 2). 언어성 소검사 중에는 '상식(t= $-2.050, \mathrm{p}=.041)$ ', '공통성 $(\mathrm{t}=-3.126, \mathrm{p}=.002)$ ', '숫자 $(\mathrm{t}=-7.869$, $\mathrm{p}<.001)^{\prime}$ 소검사 점수가 언어성 지능 소검사 항목의 총합의 평 균 점수보다 유의하게 낮았다. 동작성 소검사 중에는 '기호쓰 기 $(\mathrm{t}=-6.570, \mathrm{p}<.001)^{\prime}$ 점수가 동작성 소검사 항목의 총합의 평 균 점수보다 유의하게 낮았으며, '빠진곳 찾기( $\mathrm{t}=2.149, \mathrm{p}=.032)$, '토막짜기(t=3.749, $\mathrm{p}<.001)$ ', '모양맞추기 $(\mathrm{t}=4.322, \mathrm{p}<.001)$ '는 유의하게 높았다. 
Pearson correlation에서 $\mathrm{K}-\mathrm{CPRS}$ 는 검사연령 및 전체지 능, 언어성 지능, 동작성 지능 및 소검사들과 유의한 상관관계 를 보이지 않았다.

공존질환에 따른 집단 간 차이를 비교하였을 때, 내재화 장 애와 외현화 장애를 동반한 집단에서 공존질환이 없는 집단 과 틱장애 집단에 비해 연령이 유의하게 높았다 $(\mathrm{p}<.001)(\mathrm{Ta}-$ ble 3). 집단 간의 성별 차이는 유의미하지 않았다 $\left(\chi^{2}=3.870, p=\right.$

Table 2. Scores on $\mathrm{K}-\mathrm{W} I S C$-III in whole ADHD subjects ( $\mathrm{N}=312)$ (mean \pm SD)

\begin{tabular}{lrrr}
\hline & & \multicolumn{1}{c}{$\dagger$} & $\mathrm{P}$ \\
\hline VIQ minus PIQ & $3.5 \pm 14.5$ & 4.226 & $<.001$ \\
Subtest minus VIQ (mean) & & & \\
$\quad$ Information & $-0.2 \pm 1.7$ & -2.050 & .041 \\
Similarities & $-0.3 \pm 1.9$ & -3.126 & .002 \\
Arithmetic & $0.2 \pm 2.1$ & 1.813 & .071 \\
Vocabulary & $0.1 \pm 1.5$ & 1.589 & .113 \\
Comprehension & $0.2 \pm 2.0$ & 1.625 & .105 \\
Digit Span & $-1.4 \pm 3.1$ & -7.869 & $<.001$ \\
Subtest minus PIQ (mean) & & & \\
$\quad$ Picture completion & $0.3 \pm 2.3$ & 2.149 & .032 \\
Picture arrangement & $-0.2 \pm 2.1$ & -1.340 & .181 \\
Coding & $-1.1 \pm 2.8$ & -6.570 & $<.001$ \\
Block design & $0.5 \pm 0.5$ & 3.749 & $<.001$ \\
Object assembly & $0.5 \pm 0.5$ & 4.322 & $<.001$ \\
\hline
\end{tabular}

K-WISC-III : Korean Wechsler Intelligence Scale for Children-III, ADHD : attention-deficit hyperactivity disorder, SD : standard deviation, $\mathrm{VIQ}$ : verbal IQ, $\mathrm{PIQ}$ : performance $I Q, I O$ : intelligence quotient
.276). 이에 연령을 공변량으로 네 개의 공병집단 간 인지기능 및 $\mathrm{CPRS}$ 의 차이에 대해 MANCOVA 분석을 실시한 결과, '상 식 $(\mathrm{t}=3.554, \mathrm{p}=.008)$, '공통점 $(\mathrm{t}=4.662, \mathrm{p}=.001)$,' '어휘 $(\mathrm{t}=3.495$, $\mathrm{p}=.008)$,' 이해(t=3.723, $\mathrm{p}=.006)$, '모양맞추기 $(\mathrm{t}=4.681, \mathrm{p}=.001)$ ' 에서 집단 간 유의한 차이를 보였다. 사후검증 결과, '상식', '어 휘' 두 소검사에 대해서는 외현화 장애 집단의 점수가 다른 세 집단의 점수보다 유의하게 낮았다. '공통성', '모양맞추기' 두 소 검사에서는 공존질환이 없는 집단에서 외현화 장애 집단에 비 해 유의하게 높은 점수를 보였으며, '이해' 소검사에서는 공존 질환이 없는 집단에서 외현화 장애 집단에 비해 유의하게 점 수가 높았다.

아형별 비교에서는 각 집단 간 나이 차이는 없었다(Table 4). 성별에 있어서는 집단 간에 유의한 차이를 보였다 $\left(\chi^{2}=6.855, p=\right.$ .032). 아형별 인지기능 및 CPRS의 차이에 대해 MANOVA 분석을 실시한 결과, "이해( $\mathrm{t}=5.606, \mathrm{p}=.004)$ 와 ${ }^{\mathrm{N}} \mathrm{K}-\mathrm{CPRS}(\mathrm{t}=3.122$, $\mathrm{p}=.046)^{\prime}$ 점수에서 세 집단 간 유의한 차이를 보였다(Table 4). 사후검증 결과, '이해' 소검사 점수는 과잉행동-충동성형이 다 른 두 아형보다 유의하게 높았으며, $\mathrm{K}-\mathrm{CPRS}$ 점수는 복합형 이 부주의형보다 유의하게 높았다.

\section{고 찰}

$\mathrm{ADHD}$ 아동에서의 언어성 지능과 동작성 지능의 특성에 대 하여 Koyama 등이는 언어성 지능이 동작성 지능보다 높은 경

Table 3. Comparisons of scores on K-WISC-III according to comorbidities (mean \pm SD)

\begin{tabular}{|c|c|c|c|c|c|c|}
\hline & \multirow[b]{2}{*}{$\begin{array}{l}\text { No comorbidities }{ }^{a} \\
\qquad(N=198)\end{array}$} & \multicolumn{3}{|c|}{ Comorbidities $(\mathrm{N}=114)$} & \multirow[b]{2}{*}{$\mathrm{F}$} & \multirow[b]{2}{*}{ Post-hoc } \\
\hline & & $\begin{array}{c}\text { Internalizing } \\
\text { disorder }(\mathrm{N}=40)\end{array}$ & $\begin{array}{c}\text { Externalizing } \\
\text { disorder }^{\mathrm{c}}(\mathrm{N}=17)\end{array}$ & $\begin{array}{c}\text { Tic disorder }{ }^{d} \\
(\mathrm{~N}=34)\end{array}$ & & \\
\hline Age & $9.2 \pm 2.2$ & $11.0 \pm 2.5$ & $11.9 \pm 3.0$ & $9.8 \pm 2.2$ & $11.7^{\dagger}$ & $b, c>a, d$ \\
\hline K-CPRS & $13.4 \pm 6.0$ & $15.8 \pm 7.9$ & $17.6 \pm 5.7$ & $13.7 \pm 5.6$ & 2.384 & \\
\hline $\mathrm{FSIQ}$ & $102.3 \pm 14.1$ & $99.8 \pm 14.4$ & $95.4 \pm 13.1$ & $101.6 \pm 14.9$ & 2.782 & \\
\hline$V I Q$ & $103.6 \pm 14.4$ & $101.1 \pm 12.6$ & $94.9 \pm 14.5$ & $103.4 \pm 15.8$ & 3.331 & \\
\hline Information & $10.5 \pm 3.0$ & $10.2 \pm 2.2$ & $8.4 \pm 2.6$ & $10.1 \pm 3.0$ & $3.554^{*}$ & $a, b, d>c$ \\
\hline Similarities & $10.2 \pm 2.6$ & $9.5 \pm 2.9$ & $9.1 \pm 2.3$ & $10.1 \pm 2.7$ & $4.662^{*}$ & $a>b, c$ \\
\hline Arithmetic & $10.6 \pm 3.0$ & $10.9 \pm 2.8$ & $10.6 \pm 2.9$ & $11.4 \pm 3.5$ & 0.802 & \\
\hline Vocabulary & $10.8 \pm 2.6$ & $10.6 \pm 2.5$ & $9.2 \pm 2.7$ & $10.0 \pm 2.4$ & $3.495^{*}$ & $a, b, d>c$ \\
\hline Comprehension & $10.9 \pm 2.6$ & $10.0 \pm 3.2$ & $9.4 \pm 2.9$ & $10.5 \pm 3.2$ & $3.723^{*}$ & $a>c$ \\
\hline Digit span & $9.0 \pm 3.2$ & $9.4 \pm 3.5$ & $9.0 \pm 3.3$ & $9.6 \pm 3.5$ & 1.021 & \\
\hline $\mathrm{PIQ}$ & $99.7 \pm 14.2$ & $98.1 \pm 14.2$ & $96.5 \pm 14.1$ & $98.9 \pm 13.4$ & 1.119 & \\
\hline Picture completion & $10.3 \pm 2.9$ & $9.6 \pm 2.9$ & $9.7 \pm 3.1$ & $10.5 \pm 2.9$ & 1.177 & \\
\hline Picture arrangement & $9.8 \pm 2.6$ & $10.0 \pm 3.3$ & $8.7 \pm 2.4$ & $9.4 \pm 2.6$ & $1.091^{*}$ & \\
\hline Coding & $8.9 \pm 3.1$ & $8.9 \pm 3.1$ & $8.4 \pm 3.5$ & $8.7 \pm 3.3$ & 0.163 & \\
\hline Block design & $10.3 \pm 3.4$ & $10.8 \pm 3.6$ & $10.5 \pm 2.8$ & $10.6 \pm 3.0$ & 1.052 & \\
\hline Object assembly & $10.6 \pm 2.7$ & $9.5 \pm 3.1$ & $10.5 \pm 1.9$ & $10.1 \pm 2.7$ & 4.681 & $a>b, c$ \\
\hline
\end{tabular}

$*: p<.01, \dagger: p<.001$. K-WISC-III : Korean Wechsler Intelligence Scale for Children-III, FSIQ : Full Scale IQ, VIQ : Verbal IQ, PIQ : Performance IQ, K-CPRS : Korean-Conners' Parent Rating Scale, SD : standard deviation, IQ : intellgence quotient 
Table 4. Comparisons of scores on K-WISC-III and K-CPRS between groups (mean \pm SD)

\begin{tabular}{|c|c|c|c|c|c|}
\hline & $\begin{array}{l}\text { Inattentive type }{ }^{a} \\
(\mathrm{~N}=68)\end{array}$ & $\begin{array}{c}\text { Hyperactive-impulsive } \\
\text { type }^{\text {b }}(\mathrm{N}=33)\end{array}$ & $\begin{array}{c}\text { Combined type }{ }^{c} \\
(\mathrm{~N}=124)\end{array}$ & $\mathrm{F}$ & Post-hoc \\
\hline Age & $10.1 \pm 2.5$ & $9.2 \pm 2.3$ & $9.3 \pm 2.3$ & 2.591 & \\
\hline K-CPRS & $12.3 \pm 6.0$ & $12.4 \pm 7.0$ & $14.4 \pm 5.7$ & $3.122^{*}$ & $c>a$ \\
\hline$F S I Q$ & $99.6 \pm 14.4$ & $105.4 \pm 12.2$ & $99.2 \pm 14.0$ & 2.726 & \\
\hline$V I Q$ & $101.6 \pm 14.5$ & $106.4 \pm 11.9$ & $100.6 \pm 14.6$ & 2.171 & \\
\hline Information & $9.8 \pm 2.8$ & $11.1 \pm 2.9$ & $9.9 \pm 2.9$ & 2.471 & \\
\hline Similarities & $10.0 \pm 2.7$ & $10.0 \pm 2.2$ & $10.4 \pm 2.7$ & 0.691 & \\
\hline Arithmetic & $10.1 \pm 3.3$ & $11.5 \pm 2.2$ & $10.3 \pm 3.1$ & 2.205 & \\
\hline Vocabulary & $10.5 \pm 2.5$ & $11.2 \pm 2.3$ & $9.8 \pm 2.8$ & 1.824 & \\
\hline Comprehension & $10.9 \pm 2.9$ & $11.4 \pm 2.8$ & $9.8 \pm 2.8$ & $5.606^{\dagger}$ & $b>a, c$ \\
\hline Digit span & $8.0 \pm 2.7$ & $9.3 \pm 2.6$ & $8.4 \pm 3.3$ & 2.146 & \\
\hline$P I Q$ & $97.1 \pm 14.9$ & $102.9 \pm 14.0$ & $97.2 \pm 14.6$ & 2.177 & \\
\hline Picture completion & $10.0 \pm 2.9$ & $9.7 \pm 3.1$ & $10.4 \pm 2.8$ & 1.203 & \\
\hline Picture arrangement & $9.9 \pm 3.0$ & $10.3 \pm 3.1$ & $9.6 \pm 2.4$ & 0.884 & \\
\hline Coding & $8.5 \pm 3.0$ & $9.7 \pm 3.9$ & $8.2 \pm 3.0$ & 2.866 & \\
\hline Block design & $9.8 \pm 3.2$ & $11.3 \pm 3.5$ & $10.0 \pm 3.3$ & 2.620 & \\
\hline Object assembly & $10.1 \pm 2.9$ & $10.8 \pm 2.9$ & $10.0 \pm 2.8$ & 1.122 & \\
\hline
\end{tabular}

$*: p<.05, \dagger: p<.01$. K-WISC-III : Korean Wechsler Intelligence Scale for Children-III, FSIQ : Full Scale IQ, VIQ : Verbal IQ, PIQ : Performance IQ, K-CPRS : Korean-Conners' Parent Rating Scale, SD : standard deviation, IQ : intelligence quotient

향이 있음을 보고하였고, 지능 특성의 결과가 서양과 일본의 교육 혹은 문화적 배경의 차이를 반영하는 것일 수도 있음을 언급하면서 일본과 유사한 배경을 가진 동아시아 나라들에서 이러한 주제에 대하여 연구가 필요하다고 주장하였다. Scheirs 와 Timmers ${ }^{211}$ 의 연구에서도 유의하지는 않으나 ADHD 환아 에서 언어성 지능이 동작성 지능보다 높은 경향을 나타냈다. 국 내에서 발표된 $\mathrm{Choi}^{22)}$ 의 연구에서는 $\mathrm{ADHD}$ 집단과 정상 집단 간 전체 지능을 통제하고 언어성 지능과 동작성 지능에서 차 이를 보이는지 확인하였을 때 유의한 차이를 보이지 않았으나, 전체지능이 90 이상인 아동들의 경우 언어성 지능이 동작성 지능보다 오히려 높은 경향을 보였다. Shin 등3)의 ADHD에 관한 연구에서 언어성 지능은 동작성 지능보다 높은 결과를 보 였다. 본 연구에서도 언어성 지능이 동작성 지능보다 유의미하 게 높은 결과를 보였다. 그러나 Prifitera와 Dersh ${ }^{8}$ 의 연구에서 는 $\mathrm{ADHD}$ 환아들이 지각적 추론 요인에서 강점을 보인 반면, 언어적 이해력 요인에서 덜 발달된 모습을 보인다고 주장하였 고, $\operatorname{Kaufman}^{10)}$ 은 학습의 어려움과 관련하여 언어적 수행에 취 약점을 보이는 것 때문에 언어성 지능이 동작성 지능보다 낮다 고 설명하였다.

언어성 지능과 동작성 지능의 편차는 주로 학습장애, 비행 행동, 기질적 뇌질환 등과 관련하여 연구되어온 개념이며, 해 석 및 진단적 이용시 여러 요인을 고려해야 한다.) 예를 들면, $\mathrm{Kamphus}^{24}$ 는 언어문제, 청력의 문제, 운동능력, 성취 동기, 언어적 문화적 차이 등이 혼란변수가 될 수 있다고 하였다. 본 연구에서 나타난 언어성 지능과 동작성 지능의 편차가 Koya- $\mathrm{ma}$ 등이이 언급한대로 동서양의 문화적 차이와 관련된 것인 지, 아니면 $\mathrm{ADHD}$ 환아 집단의 이질성을 반영하는 것인지에 대해서는 다국적 연구 등 추후 연구가 필요하다.

지능검사의 소검사 항목에 대한 Prifitera와 $\mathrm{Dersh}^{8}$ 의 연구 에서는 ADHD 집단이 Arithmetic, Coding, Information, Digit Span subtests(ACID) 프로파일에서 낮은 수행을 보였다고 보고하였다. '동형 찾기(symbol search)' 소검사를 추가한 연구 결과에서는 Symbol search, Coding, Arithmetic, and Digit Span subtests(SCAD) 프로파일에서 일관되게 낮은 점수를 보이는 특성이 있다고 보고되었다. ${ }^{6,10,25)}$

Snow와 $\mathrm{Sapp}^{25}$ 도 WISC-III 소검사의 패턴이 ADHD 진단 에 있어 유용하다고 주장하였다. Shin 등 23$)$ 의 국내연구에서는 $\mathrm{ADHD}$ 환아들이 '기호 쓰기, 숫자, 이해' 소검사에서 유의미하 게 낮은 점수를 보이는 패턴을 보였으며, '산수 소검사 점수가 낮지 않은 결과에 대하여 우리 나라의 산수 과잉학습에서 기 인할 수 있다는 가능성을 설명하였다. Barkley ${ }^{1)}$ 는 ADHD를 자기-조절과 행동 억제의 문제로 설명하면서, 이러한 문제가 네 가지 집행 기능, 즉, 비언어적 작업 기억(nonverbal working memory), 언어적 작업 기억(verbal working memory), 감정/ 동기/각성의 자기-조절(self-regulation of affect/motivation/ arousal), 재구성(reconstitution)의 2차적 손상을 가져온다고 하였다. 작업 기억은 웩슬러 검사의 '숫자'나 '산수’ 같은 소검사 에서 확인될 수 있다. ${ }^{7)}$

그러나 Barkley ${ }^{1)}$ 는 몇 가지 소검사 항목들을 주의산만을 측정하는 도구로 해석하는 것은 지나친 단순화와 오도를 가 
져올 수 있다고 주장하면서, 이러한 소검사 프로파일의 진단적 이용에 대해 비판하였다. Snow와 $\mathrm{Sapp}^{25)}$ 는 이러한 논란이 구 성 타당도의 문제라기 보다는, 평가에 있어 임상가의 역할에 관한 문제라고 설명하고 있다. 본 연구에서는 $\mathrm{ADHD}$ 환아들 에게서 '상식, 공통성, 숫자, 기호쓰기' 소검사의 수행이 유의미 하게 낮은 결과를 보여, 작업 기억 등 $\mathrm{ADHD}$ 의 특성을 나타내 는 진단적 단서를 제공할 수 있을 것으로 보인다. Naglieri 등하 의 연구에서는 WISC-III와 CPRS의 상관관계가 유의미하지 않다는 결과를 보고하였으나, Conners' Teacher Rating Scale 의 Cognitive problem/Inattention 점수와 WISC-III는 의미 있는 상관관계를 보였다. Naglieri 등은 지능검사, 행동 평정, continous performance test 점수 간에 불일치가 크고 상관 관계가 적다는 연구 결과를 보고하면서, 임상에서 DMS-IV에 근거한 행동 증상과 자기-조절의 어려움과 관련된 인지적 결 핍, 두 가지 차원에 근거하여 $\mathrm{ADHD}$ 를 평가해야 함을 강조하 고 있다. Anastopolous 등 기 의 연구에서는 부모가 보고한 주 의력 산만 평정척도와 주의집중 지표가 상관관계가 없었으나, 교사가 보고한 $\mathrm{ADHD}$ 평정척도, 부주의 척도와는 상관관계 를 갖는 것으로 나타났다. 본 연구에서도 K-WISC-III와 K$\mathrm{CPRS}$ 간에는 의미 있는 상관관계가 없는 것으로 나타났는데, 이러한 결과는 $\mathrm{ADHD}$ 증상에 대한 부모 평정척도를 해석할 때 주의를 기울여야 함을 시사한다.

공존질환과 $\mathrm{ADHD}$ 환아들의 지능에 대한 기존 연구는 공 존질환의 종류와 연구자에 따라 큰 차이를 보인다. 본 연구에 서는 공존질환이 있는 집단과 공존질환이 없는 집단 간 전체 지능, 언어성 지능, 동작성 지능의 차이를 보이지 않았으나, 언 어성 지능 소검사에 해당하는 '상식', '어휘' '이해' '공통성' 항 목과 동작성 지능 소검사에 해당하는 '모양맞추기'에서 외현화 문제를 갖는집단이 유의하게 점수가 낮았다. 이는 Barkley ${ }^{2)}$ 가 $\mathrm{ADHD}$ 와 품행장애가 동반되었을 때 각 질환이 단독으로 있는 경우보다 언어성 지능이 낮다고 한 내용을 지지하는 결 과이다. Faraone 등 ${ }^{28}$ 도 품행장애와 불안장애를 동반한 경우, $\mathrm{ADHD}$ 단독으로 있는 경우보다 전체지능과 동작성 지능 소 검사인 '토막 짜기' 점수가 의미 있게 낮음을 보고하였다. Di Tra$\mathrm{ni}$ 등 ${ }^{29}$ 도 외현화 문제를 가지는 $\mathrm{ADHD}$ 환아들이 내재화 문 제를 갖는 $\mathrm{ADHD}$ 환아들보다 지능이 낮다고 보고하였다. 그 러나, Takeda 등ㄹㄱㄱㅘ Katusic 등이는 공존질환에 따른 지능의 차이는 없다고 주장하였다.

Rothenberger 등 ${ }^{311}$ 은 $\mathrm{ADHD}$ 와 틱장애가 공존할 때 $\mathrm{AD}$ $\mathrm{HD}$ 의 요인이 인지적 기능 저하 및 학습장애 등에 주로 영향 을 미치게 되며, 틱장애 요인의 영향은 분명하지 않다고 기술 하였다. Roessner 등 ${ }^{13}$ 도 $\mathrm{ADHD}$ 와 틱장애가 동반될 때 신경 심리학적 수행에 주로 영향을 미치는 것은 $\mathrm{ADHD}$ 증상이며,
이는 틱 증상과는 독립적인 것으로 보인다고 주장하였다. 본 연구에서도 틱장애가 동반된 $\mathrm{ADHD}$ 집단과 공존질환이 없 는 $\mathrm{ADHD}$ 집단 간에 지능검사에서 유의한 차이를 보이지 않 았다.

불안이나 기분 증상 같은 내재화 장애와 $\mathrm{ADHD}$ 중증도의 관련성에 대해서는 의견이 엇갈리고 있으나 ${ }^{32,33)}$ 외현화 장애가 동반된 경우는 $\mathrm{ADHD}$ 중증도가 더 높다는 연구 결과가 지배 적이다. ${ }^{12,31)}$ 본 연구에서도 외현화 문제가 있는 $\mathrm{ADHD}$ 집단이 다른 집단에 비해 높은 K-CPRS 점수를 보이고 있어 Takeda 등ㄹㄱㄱㅘ Kuhne 등 ${ }^{34}$ 의 연구들과 부분적으로 일치하는 결과를 보였으나 통계적으로 유의하지는 않았다.

또한, 본 연구에서는 공존질환 집단 간 유의한 성별 차이는 없었으며, 외현화/내재화 장애를 동반한 집단에서 공존질환이 없는 집단과 틱장애를 동반한 집단보다 연령이 높은 결과를 보였으며, 공존질환이 없는 집단이 가장 연령이 어린 결과를 보 였는데, 이는 Takeda 등ㄹㅇㅢ 이전 연구와 일치하는 결과이며, 연령이 증가할수록 공존질환의 동반이 증가하는 경향을 시사 하고 있다고 생각된다.

$\mathrm{ADHD}$ 의 아형과 관련하여 Carlson 등 ${ }^{35)}$ 의 연구에서는 과 잉행동을 동반한 attention-deficit disorder(ADD)가 과잉행 동을 동반하지 않는 $\mathrm{ADD}$ 에 비해 전체지능과 언어성 지능이 낮다고 보고하였다. 그러나 $\mathrm{Mamoto}^{36}$ 의 연구에서는 ADHD 의 부주의형과 과잉행동-충동성형/복합형 간을 비교하였을 때 WISC-III 소검사 항목의 차이는 나타나지 않았다. Di Tra$\mathrm{ni}$ 등 ${ }^{29)}$ 도 $\mathrm{ADHD}$ 아형에 따른 지능 수준의 의미 있는 차이는 없다고 보고하였다. 본 연구에서도 $\mathrm{ADHD}$ 아형에 따른 전체 지능, 언어성 지능, 동작성 지능의 차이는 나타나지 않았으나, ‘이해' 소검사 점수에서 과잉행동-충동성형이 복합형, 부주의 형보다 유의미하게 높은 결과를 보였다. 이러한 결과는 부주 의형이 포함된 ADHD 아형에서 '이해' 소검사의 수행이 저하 될 수 있음을 시사한다. '이해’ 소검사는 사회-적응적인 기능에 대한 단서를 제공하며, 유연성이 부족하거나 과도하게 구체적 인 사고를 하는 경우 점수가 낮게 나올 수 있는 항목으로, ${ }^{9}$ 본 연구 결과는 부주의형 $\mathrm{ADHD}$ 의 임상적 특성의 이해에 중요 한 정보를 제공하고 있다고 생각된다. 다만, $\mathrm{DSM}-\mathrm{V}^{37)}$ 에서는 아형을 현재 표현형(current presentations)으로 수정하고, 부 주의형의 범주에 한정된 부주의형(restrictive inattentive)을 추가하는 형태로 분류가 변화될 예정이므로 이에 대하여는 추 후 연구를 통한 확인이 필요할 것이다.

본 연구는 몇 가지 제한점이 있다. 첫째, $\mathrm{ADHD}$ 환자군과 대 조군을 비교한 연구가 아니며, 환자군의 진료기록과 심리검사 보고서를 후향적으로 분석한 연구이므로 결과 해석에 주의를 요한다. 둘째, K-WISC-III 결과 분석에 있어 언어적 이해력 지 
표, 주의집중 지표, 지각적 추론 지표, 처리속도 지표 등의 요인 지표를 포함시키지 못한 점, 코너스 평정척도 중 교사용 없이 부모용만 분석에 사용된 점 등도 이 연구의 한계점이라 하겠 다. 셋째, 공존질환 진단시 구조화된 면담도구를 사용하지 않 은 것도 후향적 연구의 한계이다. 넷째, $\mathrm{ADHD}$ 아형에 따른 결 과 분석에 있어서, 집단 간 성별의 유의한 차이를 보였으나 여 아의 비율이 적어 이를 통계적으로 통제하지 못한 점을 결과 해석시 고려해야 한다. 끝으로, 본 연구는 일 대학병원에 내원 한 환아들을 대상으로 하였으므로 이에 따르는 지리적, 사회 인구학적 한계를 고려해야 한다.

\section{결 론}

국내 일 대학병원에 방문한 $\mathrm{ADHD}$ 환아 312 명에 대한 지 능검사 결과, 언어성 지능이 동작성 지능보다 높았으며, '상식, 공통성, 숫자, 기호쓰기' 소검사의 수행이 유의하게 저하되어 있었다. 공존질환과 관련하여 외현화 문제를 갖는 집단에서 다른 집단들보다 '상식' 및 '어휘' 소검사의 점수가 낮았으며, 공 존질환이 없는 집단보다 '이해’ 소검사의 점수가 낮았다. ‘공통 성' ‘모양맞추기' 소검사에 있어 외현화 문제를 갖는 집단과 내 재화 문제를 갖는 집단에서 공존질환이 없는 집단보다 낮은 점수를 보였다. 진단적 아형 면에서는 부주의형이 포함된 아형 에서 ‘이해' 소검사의 점수가 더 낮은 결과를 보였다. 본 연구 결 과를 통해, $\mathrm{ADHD}$ 진단에 있어 지능검사가 아형 분류 및 공 존질환과의 관련성 등에 대한 평가에 보조적 기능을 할 수 있 을 것으로 판단된다.

중심 단어:ADHD · 지능검사· 공존질환 · 아형.

\section{References}

1) Barkley RA. Attention-Deficit Hyperactivity Disorder: A Handbook for Diagnosis and Treatment. 2nd ed. New York: Guilford press;1998.

2) Barkley RA. Attention-Deficit Hyperactivity Disorder: A Handbook for Diagnosis and Treatment. 3rd ed. New York: Guilford press;2006.

3) Cheon KA, Kim JH, Kang H, Kim BN, Shin D, Ahn D, et al. The Korean practice parameter for the treatment of attention-deficit hyperactivity disorder (II): diagnosis and assessment. J Korean Acad Child Adolesc Psychiatry 2007;18:10-15.

4) Jepsen JR, Fagerlund B, Mortensen EL. Do attention deficits influence IQ assessment in children and adolescents with ADHD? J Atten Disord 2009;12:551-562.

5) Bowers TG, Risser MG, Suchanec JF, Tinker DE, Ramer JC, Domoto $\mathbf{M}$. A developmental index using the Wechsler Intelligence Scale for Children: implications for the diagnosis and nature of ADHD. J Learn Disabil 1992;25:179-185, 195.

6) Assesmany A, McIntosh DE, Phelps L, Rizza MG. Discriminant validity of the WISC-III with children classified with ADHD. J Psychoeduc Assess 2001;19:137-147.

7) Prifitera A, Saklofske DH. WISC-III Clinical Use and Interpretation: Scientist-Practitioner Perspectives. San Diego: Academic Press;
1997.

8) Prifitera A, Dersh J. Base rates of WISC-III diagnostic subtest patterns among normal, learning-disabled, and ADHD samples. In: Bracken BA, McCallum RS, editors. Wechsler Intelligence Scale for Children. 3rd ed. Brendon: Clinical Psychology Publishing Co.;1993. p. 43-55.

9) Kaufman AS, Lichtenberger EO. Essentials of WISC-III and WPPSI-R Assessment. 1st ed. New York: John Wiley \& Sons;2000. p. 206-207.

10) Kaufman AS. Intelligent Testing with the WISC-III. 2nd ed. New York: John Wiley;1994.

11) Cohen M, Becker MG, Campbell R. Relationships among four methods of assessment of children with attention deficit-hyperactivity disorder. J School Psychol 1990;28:189-202.

12) Takeda T, Ambrosini PJ, deBerardinis R, Elia J. What can ADHD without comorbidity teach us about comorbidity? Res Dev Disabil 2012;33:419-425.

13) Roessner V, Becker A, Banaschewski T, Rothenberger A. Executive functions in children with chronic tic disorders with/without ADHD: new insights. Eur Child Adolesc Psychiatry 2007;16 Suppl 1:36-44.

14) Dulcan MK. Dulcan's Textbook of Child and Adolescent Psychiatry. Washington, DC: American Psychiatric Publishing Inc;2009. p.761.

15) Kwak KJ, Park HW, KIM CT. Manual for the Korean Wechsler Intelligence Scale for Children-III. Seoul: Special Education;2001.

16) Wechsler D. Manual for the Wechsler Intelligence Scale for Children (WISC-III). 3rd ed. San Antonio: Psychological Corporation;1991.

17) Conners CK. Conners Rating Scales Manual, Conner's Teacher Rating Scale, Conner's Parent Rating Scales, Instruments for use with Children and Adolescents. Toronto: Multi-Health Systems;1990.

18) Oh KJ, Lee HR. Assessment of ADHD with abbreviated Conners Rating Scale. Korean J Clin Psychol 1989;8:135-142.

19) Lee WK, Lee WH. Psychological Assessment. Seoul: Hakjisa;2012. p.103.

20) Koyama T, Tachimori H, Osada H, Kurita H. Cognitive and symptom profiles in high-functioning pervasive developmental disorder not otherwise specified and attention-deficit/hyperactivity disorder. J Autism Dev Disord 2006;36:373-380.

21) Scheirs JG, Timmers EA. Differentiating among children with PDDNOS, ADHD, and those with a combined diagnosis on the basis of WISC-III profiles. J Autism Dev Disord 2009;39:549-556.

22) Choi WS. Cognitive characteristics of children with attention deficit hyperactivity disorder reflected in K-WISC-III. Seoul: Catholic Univ.;2002.

23) Shin MS, Oh KJ, Hong KE. Cognitive characteristics of ADHD children assessed by KEDI-WISC. J Korean Acad Child Adolesc Psychiatry 1990;1:55-64.

24) Kamphus RW. Clinical Assessment of Children's Intelligence: A Handbook for Professional Practice. Boston: Allyn \& Bacon;1993.

25) Snow JB, Sapp GL. WISC-III subtest patterns of ADHD and normal samples. Psychol Rep 2000;87:759-765.

26) Naglieri JA, Goldstein S, Delauder BY, Schwebach A. Relationships between the WISC-III and the Cognitive Assessment System with Conners' rating scales and continuous performance tests. Arch Clin Neuropsychol 2005;20:385-401.

27) Anastopolous AD, Spisto MA, Maher MC. The WISC-III Freedom from Distractibility factor: Its utility in identifying children with attention deficit hyperactivity disorder. Psychol Assess 1994;6:368-371.

28) Faraone SV, Biederman J, Lehman BK, Keenan K, Norman D, Seidman LJ, et al. Evidence for the independent familial transmission of attention deficit hyperactivity disorder and learning disabilities: results from a family genetic study. Am J Psychiatry 1993;150:891895.

29) Di Trani M, Casini MP, Capuzzo F, Gentile S, Bianco G, Menghini D, et al. Executive and intellectual functions in attention-deficit/hyperactivity disorder with and without comorbidity. Brain Dev 2011; 
33:462-469.

30) Katusic MZ, Voigt RG, Colligan RC, Weaver AL, Homan KJ, Barbaresi WJ. Attention-deficit hyperactivity disorder in children with high intelligence quotient: results from a population-based study. J Dev Behav Pediatr 2011;32:103-109.

31) Rothenberger A, Roessner V, Banaschewski T, Leckman JF. Co-existence of tic disorders and attention-deficit/hyperactivity disorderrecent advances in understanding and treatment. Eur Child Adolesc Psychiatry 2007;16 Suppl 1:1-4.

32) Jensen PS, Martin D, Cantwell DP. Comorbidity in ADHD: implications for research, practice, and DSM-V. J Am Acad Child Adolesc Psychiatry 1997;36:1065-1079.

33) August GJ, Realmuto GM, MacDonald AW 3rd, Nugent SM, Crosby R. Prevalence of ADHD and comorbid disorders among elemen- tary school children screened for disruptive behavior. J Abnorm Child Psychol 1996;24:571-595.

34) Kuhne M, Schachar R, Tannock R. Impact of comorbid oppositional or conduct problems on attention-deficit hyperactivity disorder. J Am Acad Child Adolesc Psychiatry 1997;36:1715-1725.

35) Carlson CL, Lahey BB, Neeper R. Direct Assessment of the Cognitive Correlates of Attention Deficit Disorders with and Without Hyperactivity. J Psychopathol Behav Assess 1986;8:69-86.

36) Mamoto A. Cognitive processing ability of children with attentiondeficit/hyperactivity disorder, predominantly inattentive subtype. Osaka City Med J 2006;55:17-26.

37) Tannock R. Rethinking ADHD and LD in DSM-5: Proposed Changes in Diagnostic Criteria. J Learn Disabil 2013;46:5-25. 